\title{
Prostatic calculi influence the antimicrobial efficacy in men with chronic bacterial prostatitis
}

\begin{abstract}
Wei-Ping Zhao ${ }^{1}$, Yong-Tao $\mathrm{Li}^{2}$, Jun Chen ${ }^{3}$, Zhi-Gen Zhang ${ }^{1}$, Hai Jiang ${ }^{3}$, Dan Xia ${ }^{3}$, Shuo Wang ${ }^{3}$ and Ping Wang ${ }^{3}$
We studied the efficacy of culture-specific antibiotic therapy for chronic bacterial prostatitis (CBP) patients with or without prostatic calculi. This study included 101 patients (21-62 years old) who met the consensus criteria for CBP (National Institutes of Health category II). According to the results of transrectal ultrasonography (TRUS), all patients were divided into two groups: Group 1, CBP with prostatic calculi, $n=39$; Group 2, CBP without prostatic calculi, $n=62$. All patients received optimal antimicrobial therapy for 4 weeks and followed up for a minimum of 3 months (range: 3-8 months). In addition to expressed prostatic secretions (EPS) and urine culture, all patients were asked to complete the National Institutes of Health Chronic Prostatitis Symptom Index (NIH-CPSI) and the subjective global assessment (SGA). The microbiological eradication rate at the end of treatment were 32/39 (82.1\%) and 54/62

$(87.1 \%)$, while the rates for continued eradication at the end of study were $17 / 39(43.6 \%)$ and $45 / 62(72.6 \%)$ in Group 1 and Group 2 $(P<0.01)$, respectively. We observed a decrease in the total NIH-CPSI score median values from 24 to 19 in Group 1 and from 24 to 11 in Group 2. The pain subscore $(P<0.01)$, urinary sunscore $(P<0.05)$ and quality of life (QoL; $P<0.05)$ as well as the total NIH-CPSI score $(P<0.01)$ were significantly improved after antimicrobial treatment in Group 2 compared to Group 1 . Response, defined as a decrease of the NIH-CPSI total score by at least $50 \%$, was seen in Group 1 versus Group 2 in $38.5 \%$ and $58.1 \%(P<0.01)$, respectively. Our results showed that prostatic calculi influence the antimicrobial efficacy in men with $\mathrm{CBP}$. There was a noticeable decrease in the cure rate of CBP patients with prostatic calculi due to relapse after antimicrobial therapy.
\end{abstract}

Asian Journal of Andrology (2012) 14, 715-719; doi:10.1038/aja.2012.40; published online 16 July 2012

Keywords: antimicrobial drugs; chronic prostatitis; prostatic calculi

\section{INTRODUCTION}

Chronic prostatitis $(\mathrm{CP})$ represents a prevalent clinical condition that affects young and middle-aged men. It is characterized by chronic pelvic pain, urinary symptoms and an impairment in quality of life (QoL). ${ }^{1}$ The National Institutes of Health (NIH) classification of prostatitis adopted in 1995 includes several clinical categories, ranging from acute or chronic bacterial infections, chronic pelvic pain syndrome (CPPS) and even asymptomatic inflammation of the prostate. ${ }^{2}$ Category II chronic bacterial prostatitis (CBP) is characterized by recurrent episodes of symptomatic urinary tract infection mainly caused by uropathogens infecting the prostate gland and causing urological pain and/or voiding symptoms.

Stones formed in human prostate have been generally called prostatic calculi or calcareous concretions. Prostatic calculi are presumed to form by the precipitation of prostatic secretions and calcification of the corpora amylacea under inflammatory conditions. ${ }^{3,4}$ The second pathomechanism discussed in stone formation is that intraprostatic reflux may cause chemical prostatitis. Some studies showing many constituents of prostatic calculi could be found only in urine, not in prostatic secretions. ${ }^{5-7}$

Prostatic calculi are common in men evaluated for prostate cancer or benign prostatic hyperplasia, but the significance of prostatic calculi with respect to urological diseases and symptoms is obscure. ${ }^{8,9}$
Prostatic calculi are very common in prostatitis, and one study indicated that prostatic calculi were found in $59 \%$ of the patients and only $1 \%$ of the controls $(P<0.001)$, confirming their specific relation to prostate inflammation. ${ }^{10}$ It has been reported that a prostatic calculus is actually a cluster of bacteria and that these calculi may be the cause of prolonged bacteriosis in patients with recurrent urinary tract infections. ${ }^{11}$ However, it is unclear whether prostatic calculi influence the efficiency of antimicrobial therapy in patients with CP.

In the present study, we aimed to examine, through a pilot study, the impact of prostatic calculi on the efficiency of antimicrobial therapy in men with CBP (NIH category II).

\section{MATERIALS AND METHODS}

From March 2008 to October 2010, 106 men with a clinical diagnosis of CBP were selected from a population of 1258 patients with suspected CP. Before their inclusion in the study, all patients provided written informed consent.

Men were eligible for the study if their age was at least 18 years, and they reported symptoms of voiding or pain in the pelvic region for at least 3 months during the 6 months before entry. The exclusion criteria were the presence of a history of epididymitis or sexually transmitted disease; treatment with antimicrobial substances penetration into 
prostatic 4 weeks prior to the study entry; residual urine volume $>50 \mathrm{ml}$ resulting from bladder outlet obstruction; cancer of the genitourinary tract; a history of intravesical chemotherapy; active urethral stricture; a history of pelvic radiation or systemic chemotherapy; neurological disease affecting the bladder; prostate or bladder surgery; inflammatory bowel disease; liver function test values (SGOT/AST, SGPT/ALT or total bilirubin) twice greater than the upper limit of normal; and serum creatinine $0.5 \mathrm{mg}$ per $100 \mathrm{ml}$ above the upper limit of normal or a calculated serum creatinine clearance of less than $30 \mathrm{ml} \mathrm{min}^{-1}$.

All patients had a complete history, physical examination and transrectal ultrasonography (TRUS) of the prostate (Figure 1). The bacteriological diagnosis was confirmed by the Meares-Stamey four-glass lower urinary tract localization test ${ }^{12}$ using the following criteria: (i) if the bacterial colony forming units (CFUs) of the cultured expressed prostatic secretion (EPS) obtained by rectal digital prostatic massage or, if sufficient EPS could not be obtained, the CFU of the cultured voided bladder urine after prostatic massage (VB3) was $\geqslant 10^{3} \mathrm{CFU} \mathrm{ml} \mathrm{m}^{-1}$ and the leucocyte was $\geqslant 10$ per high-power field $(\mathrm{HPF} \times 400)$ in EPS or VB3, respectively; and (ii) a 10-fold increase in CFU in EPS and urine after prostate massage (VB3) compared with first voided urine (VB1) and midstream urine (VB2). All colony counts were reported for any bacteria isolated and drug sensitivity tests of bacteria were performed. The bacteriological response was based on the results of the appropriate cultures taken before, after therapy and the end of study.

Symptoms were quantified by the National Institute of Health Chronic Prostatitis Symptom Index (NIH-CPSI). ${ }^{13}$ The NIH-CPSI consists of nine questions, exploring the three major domains of prostatitis, i.e., pain (scored 0-21), voiding disturbances (scored 0-10) and QoL impact (scored 0-12); the total NIH-CPSI score is 0-43. All patients were asked to complete the NIH-CPSI questionnaire before treatment and the end of study.

To distinguish treatment responders from non-responders, patients with more than 50\% improvement in their total NIH-CPSI score were classified as responders, and patients with $25 \%-50 \%$ improvement in their NIH-CPSI total scores were classified as partial responders. Patients with less than $25 \%$ improvement were classified as poor responders. $^{14,15}$

In addition, a subjective global assessment (SGA) was completed. ${ }^{16-19}$ With SGA, the patient's improvement was graded as none $(<25 \%$ improvement), mild (25\%-50\% improvement), moderate (50\%-75\% improvement) or marked improvement $(>75 \%)$. Responders were

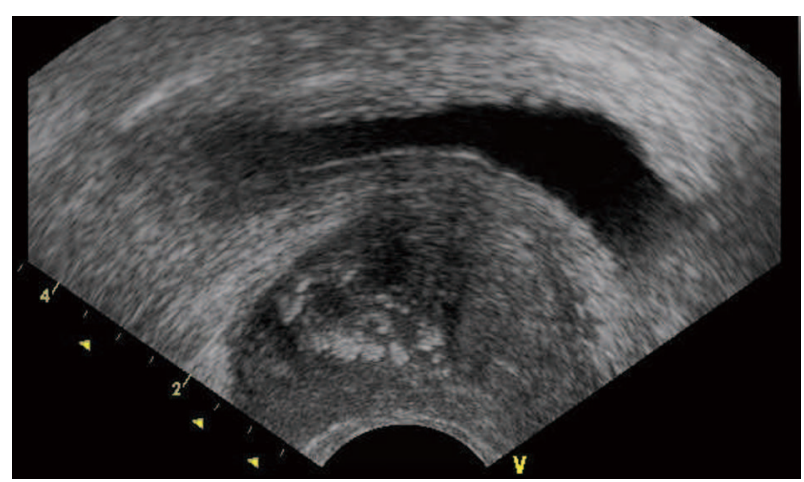

Figure 1 Typical TRUS appearance of prostatic calculi of the patients described in this study. TRUS, transrectal ultrasonography. predefined as those who indicated that they had a moderate or marked improvement in their global symptoms.

Patients were divided into two groups according to the results of TRUS of the prostate: Group 1, CBP with prostatic calculi; Group 2, CBP without prostatic calculi. Hyperechoic areas without shadowing and tiny stippled calcifications (less than $3 \mathrm{~mm}$ ) were not considered as prostatic calculi for the purpose of this study. The duration of total therapy in this clinical study was 4 weeks. All patients received optimal antimicrobial therapy according to the results of their culture and sensitivity test.

The follow-up schema included clinical examinations and the questionnaire-based reevaluation of NIH-CPSI and SGA at 1, 3, 5, 8 and 12 months after treatment. Any patient receiving the drug for less than 28 days or follow-up period less than 1 month was considered as a dropout. Eradication was defined as causative organisms absent $\left(<10^{3} \mathrm{CFU} \mathrm{m} \mathrm{^{-1 } )}\right.$ at the end of therapy. Continued eradication was defined as causative organisms absent $\left(<10^{3} \mathrm{CFU} \mathrm{ml}^{-1}\right)$ after completion of therapy and during the follow-up period; Relapse was defined as causative organisms absent $\left(<10^{3} \mathrm{CFU} \mathrm{ml}^{-1}\right)$ after completion of therapy but recurrence of the same organisms during the follow-up period at $\geqslant 10^{3} \mathrm{CFU} \mathrm{ml}^{-1}$; Superinfection was defined as causative organism absent $\left(<10^{3} \mathrm{CFU} \mathrm{\textrm {ml } ^ { - 1 } )}\right.$ after completion of therapy but appearance of another infecting organism at the followup period at $\geqslant 10^{3} \mathrm{CFU} \mathrm{ml} \mathrm{m}^{-1}$.

Statistical analysis was performed using SPSS, version 11.0 (SPSS Inc., Chicago, IL, USA). Ordinal scale values were compared with the Mann-Whitney-Wilcoxon test, and categorical variables were compared with the chi-square test. Spearman's rank correlation coefficient was used as a nonparametric test to assess the linear correlation between variables. The parametric variables are reported as median values (range). The criterion for statistical significance was $P<0.05$.

\section{RESULTS}

The mean age of the 106 CBP patients included in this study is 37.6 years (range: $21-62$ years). TRUS showed that 41 (38.7\%) of them had significant calculi within the prostate. Five men failed to complete the 4-week treatment because of drug side effects, including two in Group 1 and three in Group 2. They were excluded from further analysis. Patients were followed up for a minimum of 3 months (range: 3-8 months). Follow-up data were obtained on 101 men at the end of the study (39 in Group 1 and 62 in Group 2).

Patients were assessed for pathogens at the start of treatment (baseline), at the end of antimicrobial treatment and the end of study. As shown in Table 1, main bacterial strains include: Gram-negative bacteria (Escherichia coli, Pseudomonas aeruginosa, Pseudomonas spp.) and Gram-positive bacteria (Enterococcus faecalis, Staphylococcus aureus, Staphylococcus haemolyticus, Staphylococcus epidermidis, Staphylococcus warneri). Antibiotics chosen on the basis of sensitivities included fluoroquinolones, Co-Trimoxazole and macrolides. The therapeutic protocols are shown below: levofloxacin $500 \mathrm{mg}$ daily; ciprofloxacin $250 \mathrm{mg}$ twice daily; Co-Trimoxazole (80/400 mg) two tablets twice daily; and roxithromycin $150 \mathrm{mg}$ twice daily. The duration of total therapy in this clinical study was 28 days.

Table 1 also shows the post-therapy eradication rates, continued eradication rates, relapse rates and superinfection rates for frequently isolated pathogens. At the end of treatment (4 weeks) visit, the bacteriological response was eradication in 32 of 39 (82.1\%) patients in Group 1; the remaining seven patients $(17.9 \%)$ had a persistent infection. The microbiological eradication rate was $87.1 \%$ in Group 2. At 
Table 1 The microbiologic response for each isolated pathogen

\begin{tabular}{|c|c|c|c|c|c|c|c|c|c|c|}
\hline \multirow[b]{2}{*}{ Pathogens } & \multicolumn{5}{|c|}{ Group $1(n=39)$} & \multicolumn{5}{|c|}{ Group $2(n=62)$} \\
\hline & $n$ & $\begin{array}{l}\text { Eradication } \\
(\%)\end{array}$ & $\begin{array}{l}\text { Continued } \\
\text { eradication (\%) }\end{array}$ & $\begin{array}{l}\text { Relapse } \\
\text { (\%) }\end{array}$ & $\begin{array}{l}\text { Superinfection } \\
\text { (\%) }\end{array}$ & $n$ & $\begin{array}{l}\text { Eradication } \\
\text { (\%) }\end{array}$ & $\begin{array}{l}\text { Continued } \\
\text { eradication (\%) }\end{array}$ & $\begin{array}{l}\text { Relapse } \\
\text { (\%) }\end{array}$ & $\begin{array}{l}\text { Superinfection } \\
\text { (\%) }\end{array}$ \\
\hline $\begin{array}{c}\text { Pseudomonas } \\
\text { aeruginosa }\end{array}$ & 2 & $1(50.0)$ & $1(50.0)$ & $0(0.0)$ & $0(0.0)$ & 5 & $3(60.0)$ & $2(40.0)$ & $0(0.0)$ & $1(20.0)$ \\
\hline $\begin{array}{l}\text { Pseudomonas } \\
\text { spp. }\end{array}$ & 4 & $2(50.0)$ & $1(25.0)$ & $1(25.0)$ & $0(0.0)$ & 3 & $2(66.7)$ & $2(66.7)$ & $0(0.0)$ & $0(0.0)$ \\
\hline $\begin{array}{l}\text { Staphylococcus } \\
\text { aureus }\end{array}$ & 5 & $3(60.0)$ & $1(20.0)$ & $1(20.0)$ & $1(20.0)$ & 7 & $5(71.4)$ & $3(42.9)$ & $1(14.3)$ & $1(14.3)$ \\
\hline $\begin{array}{c}\text { Staphylococcus } \\
\text { haemolyticus }\end{array}$ & 4 & $4(100.0)$ & $2(50.0)$ & $2(50.0)$ & $0(0.0)$ & 3 & $2(66.7)$ & $1(33.3)$ & $0(0.0)$ & $1(33.3)$ \\
\hline $\begin{array}{l}\text { Staphylococcus } \\
\text { epidermidis }\end{array}$ & 3 & $3(100.0)$ & $3(100.0)$ & $0(0.0)$ & $0(0.0)$ & 4 & $4(100.0)$ & $0(0.0)$ & $0(0.0)$ & $0(0.0)$ \\
\hline
\end{tabular}

Group 1: chronic bacterial prostatitis with prostatic calculi; Group 2: chronic bacterial prostatitis without prostatic calculi; - :no corresponding item.

Data presented as number of patients, with percentages in parentheses.

$* P<0.01$, vs. Group 2.

the end of the study, the bacteriological response was continued eradication in 45 patients (72.6\%) in Group 2, while five patients $(8.1 \%)$ had a recurrence of his original pathogen and four patients $(6.5 \%)$ had become infected with a new pathogen. However, the response was continued eradication only in 17 of 39 patients (43.6\%) in Group 1, while relapse and superinfection were found in $12(30.8 \%)$ and 3 patients $(7.7 \%)$, respectively. There were statistically significant differences in continued eradication and relapse rate between Group 1 and Group 2.

In our series, the follow-up ranged between 3 and 8 months. As shown in Table 2, at 3-5 months after therapy, one patient in Group 1 and five patients in Group 2 were lost to follow-up. At 6-8 months after therapy, four patients in Group 1 and nine patients in Group 2 were lost to follow-up. We analyze the eradication data on the basis of the time lag between the end of therapy and the end of the follow-up period. There were statistically significant differences in eradication rate between Group 1 and Group 2 at 3-5 months and 6-8 months of follow-up phase, respectively.

There were no significant differences between the two groups with regard to the total NIH-CPSI score and subscore before treatment. As shown in Table 3, at the end of study, there were significant differences in total score $(P<0.01)$, pain subscore $(P<0.01)$, urinary subscore
$(P<0.05)$ and QoL subscore $(P<0.05)$ between Group 1 and Group 2. We also analyzed the relationship between eradication of bacteria and symptom improvement. There were significant symptom improvement (via NIH-CPSI score) in the successfully treated patients (microbiological eradication by patient) compared with unsuccessfully treated patients.

As shown in Figure 2, the number of patients rated as responders (more than 50\% improvement in their total NIH-CPSI score) was significantly different between Group 1 and Group 2 at the end of the study $(38.5 \%$ vs. $58.1 \%, P<0.01)$. Similarly, a significantly greater percentage of patients in Group 2 demonstrated a 50\% improvement in SGA compared to Group 1 ( $45.2 \%$ vs. $28.2 \%$, for a $\geqslant 50 \%$ SGA improvement) at the end of the study. In CBP patients with or without prostatic calculi, no correlation was found between the type of organisms isolated and the response to therapy.

\section{DISCUSSION}

Prostatic calculi are common in urological practice. They are associated with chronic inflammation, epithelial damage and obstruction of the glandular tissue on histological examination; ${ }^{20}$ however, their clinical significance remains unclear. There may be different incidences of prostatic calculi with diverse definition and community.

Table 2 The pathogen eradication data on the basis of the time lag between the end of therapy and the end of the follow-up period

\begin{tabular}{|c|c|c|c|c|c|c|c|c|c|c|c|c|}
\hline \multirow{3}{*}{ Time } & \multicolumn{4}{|c|}{ End of treatment } & \multicolumn{4}{|c|}{ 3-5 months } & \multicolumn{4}{|c|}{ 6-8 months } \\
\hline & \multicolumn{2}{|c|}{ Group 1} & \multicolumn{2}{|c|}{ Group 2} & \multicolumn{2}{|c|}{ Group 1} & \multicolumn{2}{|c|}{ Group 2} & \multicolumn{2}{|c|}{ Group 1} & \multicolumn{2}{|c|}{ Group 2} \\
\hline & $n$ & $\%$ & $\mathrm{n}$ & $\%$ & $n$ & $\%$ & $n$ & $\%$ & $n$ & $\%$ & $n$ & $\%$ \\
\hline No. of evaluated patients & 39 & 100 & 62 & 100 & 38 & 97.4 & 57 & 91.9 & 35 & 89.7 & 53 & 85.5 \\
\hline Eradication & 32 & 82.0 & 54 & 87.1 & 20 & $51.3 *$ & 46 & 74.2 & 17 & $43.6 *$ & 45 & 72.6 \\
\hline Relapse & - & & - & & 9 & & 5 & & 3 & & - & \\
\hline Superinfection & - & & - & & 3 & & 3 & & 0 & & 1 & \\
\hline Lost to follow-up & - & & - & & 1 & & 5 & & 4 & & 9 & \\
\hline
\end{tabular}

Group 1: chronic bacterial prostatitis with prostatic calculi; Group 2: chronic bacterial prostatitis without prostatic calculi; —: no corresponding item.

$* P<0.01$, vs. Group 2. 
Table 3 The results of NIH-CPSI of all patients

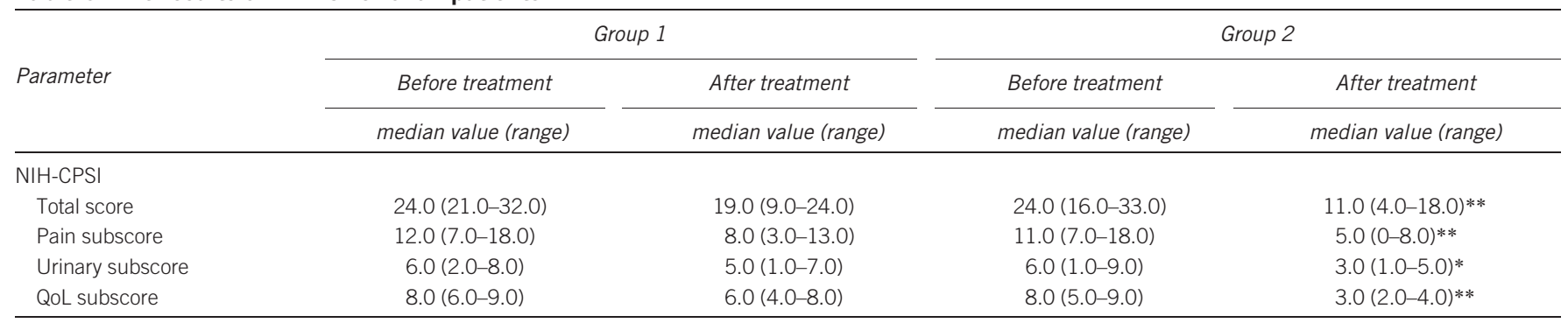

Abbreviations: NIH-CPSI, National Institute of Health Chronic Prostatitis Symptom Index; QoL, quality of life.

Group 1: chronic bacterial prostatitis with prostatic calculi; Group 2: chronic bacterial prostatitis without prostatic calculi.

Data presented as median values (range).

$* P>0.05, * * P<0.01$, vs. Group 1 .

In one study of 47 TRUS scans for men with CP/CPPS, prostatic calculus was seen in $47 \%$ patients. This prostatic calculus was associated with inflammation, positive bacterial cultures and longer symptom duration but not symptom severity. The investigators concluded that calculus might be a disease marker and potential therapeutic target in men with CPPS. ${ }^{21}$ In our series, 106 CBP patients were evaluated with TRUS, the incidence of prostatic calculus was $38.7 \%$. However, there was no significant difference in chronic prostatitis symptom scores (total and all subdomains) between patients with CBP with and without prostatic calculi.

One study found that there is no difference in rates of localization of bacterial cultures for men with CP/CPPS compared to the asymptomatic control population. ${ }^{22}$ However, there is little debate concerning the necessity of treating CBP where a typical pathogen is isolated from the site of infection. Because of suitable pharmacokinetic characteristics, good penetration into the prostate and a wide antibacterial spectrum, sulfonamides, macrolides and fluoroquinolones are the drugs of choice for CBP patients. According to some studies, the cure rate after fluoroquinolone application ranges from $63 \%$ to $86 \% .{ }^{23,24}$ Unfortunately, there is no real acceptance as to when therapeutic trials meet the criteria of a sufficient follow-up so as to give proven evidence of microbial eradication. The present study was designed to compare the antimicrobial therapeutic efficacy between CBP with prostatic calculi and without prostatic calculi. After 4 weeks of antimicrobial treatment, $82.1 \%$ of the patients with prostatic calculi and $87.1 \%$ of the patients without prostatic calculi in the microbiologically assessable population experienced eradication of all pathogens present at

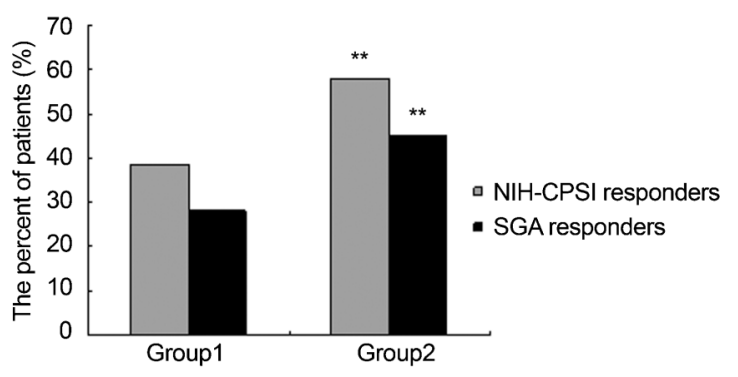

Figure 2 Clinical response rates after antimicrobial therapy in men with CBP. Group 1: CBP with prostatic calculi; Group 2: CBP without prostatic calculi. NIHCPSI responders: patients with more than 50\% improvement in their total $\mathrm{NIH}$ CPSI score. SGA responders: patients with more than $50 \%$ improvement in their SGA. CBP, chronic bacterial prostatitis; NIH-CPSI, National Institute of Health Chronic Prostatitis Symptom Index; SGA, subjective global assessment. $* * P<0.01$, vs. Group 1. study entry. However, at the end of the study visit, the continued eradication rates in patients with calculi and without calculi were $43.6 \%$ and $72.6 \%(P<0.01)$, respectively. Our results support the hypothesis that prostate calculi may hamper antibiotic therapy; ${ }^{25}$ in our series, sonographic evidence of prostate calculi was a predictive parameter for the outcome of antimicrobial therapy.

Our results also indicated that a noticeable decrease in the cure rate of CBP patients with prostatic calculi was attributed to relapse after antibiotic therapy. Explanations for this phenomenon may be associated with the persistence of a protected site, such as a biofilm. The majority of bacteria grow in biofilms, which is an agglomeration of microorganisms (forming microcolonies) and their extracellular polysaccharide matrix products. ${ }^{26-28}$ Bacterial biofilm is difficult to eradicate because of its resistance to antimicrobial treatment and removal by the host immune system. ${ }^{29}$ A recent study proves that bacterial strains able to produce biofilms consistently are present in CBP. ${ }^{30}$ Additionally, prostatic calcifications are biofilm-related. That study also indicated that the presence of bacteria can be detected by electron microscopy in prostatic calculi in two different ways. Firstly, bacteria may seem to be an essential constituent of the calculi with a sort of repetitive globular unit forming the calculi itself. Secondly, bacteria may seem to colonize the surface of the previously formed calculi. Based on our clinical results and data mentioned above, we believed that biofilms influence the antimicrobial therapeutic efficacy in CBP patients with prostatic calculi. Moreover, A small fraction of bacteria within biofilms are dormant and escape from the effects of antibiotics because of their very low metabolic activity, these dormant bacteria are able to induce recurrence of the infection in chronic cystitis. ${ }^{31}$ These dormant bacteria may be a possible origin of the relapse in CBP patients.

As CBP often relapses, a long-term follow-up of at least 6 months is desirable. One of the limitations of this study was absence of long-term follow-up. As antimicrobial drugs were given for 4 weeks, we do not know the efficacy of a longer duration of treatment. Moreover, in our series, selection of antimicroal drugs is based on the results of their culture and sensitivity test. There are some differences between antimicroal drugs in men with and without prostatic calculi; therefore, there is a potential bias to the study.

\section{CONCLUSIONS}

The results of this trial demonstrate that prostatic calculi influence the antimicrobial therapeutic efficacy in men with CBP. After 4 weeks of antimicrobial therapy and follow-up, a greater percentage of organisms were continuously eradicated in patients without prostatic calculi $(72.6 \%)$ than with prostatic calculi $(43.6 \% ; P<0.01)$. Similarly, patients 
without prostatic calculi resulted in a significantly higher symptom improvement compared to those with prostatic calculi.

\section{AUTHOR CONTRIBUTIONS}

The work presented here was carried out under collaboration of all authors. WPZ and PW designed the study, carried out the study, followed up for patients, analyzed the data, interpreted the results and participated in drafting and revising the paper. YTL made substantial contributions to the conception and design of the study and assessment the pathogens and the choice of antimicrobial drugs. JC, ZGZ, HJ, DX and SW participated in patient screening and assessment. All authors read and approved the final manuscript.

\section{COMPETING FINANCIAL INTERESTS}

The authors declare that they have no competing financial interests.

\section{ACKNOWLEDGMENTS}

This study supported by National Natural Science Foundation of China (No. 30973002), Zhejiang Provincial Natural Science Foundation of China (No. Y2090099) and Foundation of Science Technology Department of Zhejiang Province (No. 2009C33041).

1 Schaeffer AJ. Clinical practice. Chronic prostatitis and the chronic pelvic pain syndrome. N Engl J Med 2006; 355: 1690-8.

2 Krieger JN, Nyberg L Jr, Nickel JC. NIH consensus definition and classification of prostatitis. JAMA 1999; 282: 236-7.

3 Smith V. Prostatic corpora amylacea and their calcification. Surg Forum 1965; 16 : 501-2.

4 Sfanos KS, Wilson BA, de Marzoa AM, Isaacs WB. Acute inflammatory proteins constitute the organic matrix of prostatic corpora amylacea and calculi in men with prostate cancer. Proc Natl Acad Sci USA 2009; 106: 3443-8.

5 Lee SE, Ku JH, Park HK, Kwak C, Jeong H et al. Prostatic calculi do not influence the level of serum prostate specific antigen in men without clinically detectable prostate cancer and prostatitis. J Urol 2003; 170: 745-8.

6 Sutor DJ, Wooley SE. The crystalline composition of prostatic calculi. Br J Urol 1974 46: 533-5.

7 Torres Ramirez C, Aguilar Ruiz J, Zuluaga Gomez A, Espuela Orgaz R, del Rio Samper S. A crystallographic study of prostatic calculi. J Urol 1980; 124: 840-3.

8 Park SW, Nam JK, Lee SD, Chung MK. Are prostatic calculi independent predictive factors of lower urinary tract symptoms? Asian J Androl 2010; 12: 221-6.

9 Hwang EC, Choi HS, Im CM, Jung SI, Kim SO et al. Prostate calculi in cancer and BPH in a cohort of Korean men: presence of calculi did not correlate with cancer risk. Asian $J$ Androl 2010; 12: 215-20.

10 Bartoletti R, Cai T, Mondaini N, Dinelli N, Pinzi N et al. Prevalence, incidence estimation, risk factors and characterization of chronic prostatitis/chronic pelvic pain syndrome in urological hospital outpatients in Italy: results of a multicenter case-control observational study. J Urol 2007; 178: 2411-5.

11 Eykyn S, Bultitude MI, Mayo ME, Lloyd-Davies RW. Prostatic calculi as a source of recurrent bacteriuria in the male. Br J Urol 1974; 46: 527-32.

12 Meares EM, Stamey TA. Bacteriologic localization patterns in bacterial prostatitis and urethritis. Invest Urol 1968; 5: 492-518.

13 Litwin MS, McNaughton-Collins M, Fowler FJ Jr, Nickel JC, Calhoun EA et al. The National Institutes of Health chronic prostatitis symptom index: development and validation of a new outcome measure. J Urol 1999; 162: 369-75.

14 Propert KJ, Litwin MS, Wang Y, Alexander RB, Calhoun E et al. Responsiveness of the National Institutes of Health Chronic Prostatitis Symptom Index (NIH-CPSI). Qual Life Res 2006; 15: 299-305.

15 Nickel JC. Treatment of chronic prostatitis/chronic pelvic pain syndrome. Int J Antimicrob Agents 2008; 31 (Suppl 1): S112-6.

16 Nickel JC, Sorensen R. Transurethral microwave thermotherapy for nonbacteria prostatitis: a randomized doubleblind sham controlled study using new prostatitis specific assessment questionnaires. J Urol 1996; 155: 1950-4.

17 Nickel JC, Pontari M, Moon T, Gittelman M, Malek G et al. A randomized, placebo controlled, multi-multicenter study to evaluate the safety and efficacy of rofecoxib in the treatment of chronic nonbacterial prostatitis. J Urol 2003; 169: 1401-5.

18 Propert KJ, Alexander RB, Nickel JC, Kusek JW, Litwin MS et al. Design of a multicenter randomized clinical trial for chronic prostatitis/chronic pelvic pain syndrome. Urology 2002; 59: 870-6.

19 Chen R, Nickel JC. Acupuncture ameliorates symptoms in men with chronic prostatitis/ chronic pelvic pain syndrome. Urology 2003; 61: 1156-9.

20 de Marzo AM, Platz EA, Sutcliffe S, Xu J, Grönberg H et al. Inflammation in prostate carcinogenesis. Nat Rev Cancer 2007; 7: 256-69.

21 Shoskes DA, Lee CT, Murphy D, Kefer J, Wood HM. Incidence and significance of prostatic stones in men with chronic prostatitis/chronic pelvic pain syndrome. Urology 2007; 70: 235-8.

22 Nickel JC, Alexander RB, Schaeffer AJ, Landis JR, Knauss JS et al. Leukocytes and bacteria in men with chronic prostatitis/chronic pelvic pain syndrome compared to asymptomatic controls. J Urol 2003; 170: 818-22.

23 Wagenlehner FM, Diemer T, Naber KG, Weidner W. Chronic bacterial prostatitis (NIH type II): diagnosis, therapy and influence on the fertility status. Andrologia 2008; 40: 100-4.

24 Bundrick W, Heron SP, Ray P, Schiff WM, Tennenberg AM et al. Levofloxacin versus ciprofloxacin in the treatment of chronic bacterial prostatitis: a randomized doubleblind multicenter study. Urology 2003; 62: 537-41.

25 Meares EM Jr. Infection stones of prostate gland. Laboratory diagnosis and clinical management. Urology 1974; 4: 560-6.

26 Sutherland IW, Hughes KA, Skillman LC, Tait K. The interaction of phage and biofilms. FEMS Microbiol Lett 2004; 232: 1-6.

27 Tenke P, Riedl CR, Jones GL, Williams GJ, Stickler D et al. Bacterial biofilm formation on urologic devices and heparin coating as preventive strategy. Int J Antimicrob Agents 2004; 23 (Suppl 1): S67-74.

28 Nickel JC, Downey J, Clark J, Ceri H, Olson M. Antibiotic pharmacokinetics in the inflamed prostate. J Urol 1995; 153: 527-9.

29 Donlan RM. Preventing biofilms of clinically relevant organisms using bacteriophage. Trends Microbiol 2009; 17: 66-72.

30 Mazzoli S. Biofilms in chronic bacterial prostatitis (NIH-II) and in prostatic calcifications. FEMS Immunol Med Microbiol 2010; 59: 337-44.

31 Opal SM. Communal living by bacteria and the pathogenesis of urinary tract infections. PLoS Med 2007; 4: e349. 